\title{
Engineering the Dissipation of Crystalline Micromechanical Resonators
}

\author{
Erick Romero $\odot,{ }^{1}, *$ Victor M. Valenzuela $\odot,{ }^{2}$ Atieh R. Kermany, ${ }^{1}$ Leo Sementilli, ${ }^{1}$ Francesca Iacopi $\odot,{ }^{3}$ \\ and Warwick P. Bowen ${ }^{1}$ \\ ${ }^{1}$ Centre for Engineered Quantum Systems, School of Mathematics and Physics, The University of Queensland, \\ Australia \\ ${ }^{2}$ Facultad de Ciencias Físico-Matemáticas, Universidad Autónoma de Sinaloa, Mexico \\ ${ }^{3}$ School of Electrical and Data Engineering, University of Technology Sydney, NSW, Australia
}

(Received 6 November 2019; revised manuscript received 19 February 2020; accepted 13 March 2020; published 3 April 2020)

\begin{abstract}
High-quality micro- and nanomechanical resonators are widely used in sensing, communications, and timing, and have future applications in quantum technologies and fundamental studies of quantum physics. Crystalline thin films are particularly attractive for such resonators due to their prospects for high quality, high intrinsic stress, high yield strength, and low dissipation. However, when such films are grown on a silicon substrate, interfacial defects arising from lattice mismatch with the substrate have been postulated to introduce additional dissipation. Here, we develop a back-side etching process for single-crystal silicon carbide microresonators that allows us to quantitatively verify this prediction. By engineering the geometry of the resonators and removing the defective interfacial layer, we achieve quality factors exceeding a million in silicon carbide trampoline resonators at room temperature, a factor of five higher than those achieved without removal of the interfacial defect layer. We predict that similar devices fabricated from ultrahigh-purity silicon carbide, leveraging its high yield strength, could enable room-temperature quality factors as high as $6 \times 10^{9}$.
\end{abstract}

DOI: 10.1103/PhysRevApplied.13.044007

\section{INTRODUCTION}

Micro- and nanomechanical resonators have a wide range of applications in industry and fundamental science, ranging from precision sensing of mass [1], single molecules [2], ultrasound [3], magnetic fields [4], and inertia [5], to tests of spontaneous-collapse models in quantum mechanics [6,7] and memories and interfaces for quantum computers $[8,9]$. Achieving a high resonator quality factor is critical for many of these applications.

Recently, remarkable progress has been made in improving the quality factor of micro- and nanomechanical resonators fabricated from highly stressed thin amorphous films - most particularly amorphous silicon nitride — on a silicon substrate [10-15]. This progress has been achieved through a combination of dissipation engineering [12], to decrease both external energy loss to the environment and internal material losses, and strain engineering, to approach the material yield strength and thereby dilute the dissipation $[15,16]$. However, these strategies are now approaching their limits for amorphous materials.

Crystalline materials offer a range of advantages that could allow them to go beyond these limits. High-purity crystalline materials have a lower density of defects than amorphous materials, allowing significantly higher

*e.romero@uq.edu.au intrinsic quality factors. For instance, intrinsic quality factors above $10^{5}$ have been reported for highly pure diamond [17,18], calcium fluoride [19], and silicon carbide [20], which has exhibited quality factors higher than $10^{6}$ when surface losses are eliminated [21]. This compares with values of 25000 in amorphous silicon nitride [22] and 1000 in amorphous silicon [23]. Furthermore, due to crystal-lattice mismatch, crystalline materials can be grown with a high intrinsic stress [24], which is crucial for dissipation dilution. Strained single-crystal string resonators have been reported with quality factors exceeding $10^{5}$ for $\mathrm{Ga}(\mathrm{N}, \mathrm{As})$ and $10^{6}$ for $\mathrm{SiC}[25,26]$, while $\mathrm{GaAs}$ and $\mathrm{In}_{x} \mathrm{Ga}_{1-x} \mathrm{P}$ nanomembranes have reached quality factors above $10^{6}[27,28]$. Moreover, crystalline materials have a high yield strength, increasing their potential for applications using both dissipation and strain engineering. For instance, crystalline silicon carbide thin films can exhibit an intrinsic stress as high as $1.5 \mathrm{GPa}$ [29] and have a yield strength of $21 \mathrm{GPa}$ [30]. This compares with values of 1.3 and $6 \mathrm{GPa}$ for amorphous silicon nitride. However, even with these significant advantages, thin-film crystalline resonators have not seen the same dramatic improvements in quality as their amorphous counterparts. This is due in part to the increased complexity of fabrication [18,27,28], and - when grown on a silicon substrate - in part to the presence of dislocations and a high density of stacking faults in close proximity to the interface, which have 
been postulated to degrade the mechanical quality factor [31-34].

Here, we develop a back-side etching technique for the fabrication of crystalline thin-film resonators. We use this to fabricate high-quality single-crystal silicon carbide trampoline resonators, and to characterize the effect of the interfacial defect layer on their mechanical quality factor. By measuring trampoline resonators of varying thicknesses, both back- and front-side etched, we are able to build a quantitative model that allows us to extract the volumetric intrinsic quality factors of the interfacial layer and of the high-quality silicon carbide far from the interface, as well as the intrinsic surface quality factor. We find that defects degrade the volumetric quality factor by more than an order of magnitude near the interface, and we achieve a diluted quality factor exceeding one million in devices in which the interfacial defect layer is removed. Our model predicts that diluted quality factors as high as $6 \times 10^{9}$ may be possible using both dissipation and strain engineering in high-purity single-crystal silicon carbide.

\section{DEVICE DESIGN AND FABRICATION}

Our trampoline design features a square inner island of side length $40 \mu \mathrm{m}$, suspended within a $700 \mu \mathrm{m} \times 700 \mu \mathrm{m}$ square hollow by four tethers $5 \mu \mathrm{m}$ wide and approximately $500 \mu \mathrm{m}$ long. These are connected to the substrate by adiabatically widened and rounded clamping points with a radius of curvature $R$, as shown in the inset of Fig. 1(a). In contrast to clamp-tapered approaches, where dissipation dilution is achieved through localized stress [35], the dissipation dilution present in our trampolines comes from clamping points with a radius of curvature engineered to optimize the fraction of elastic energy stored as elongation as opposed to bending $[12,36]$. In this approach, two counteracting and competing mechanisms are present: the increased material volume at the widened clamps stores a larger amount of bending energy, while the increased rigidity reduces the overall bending $[37,38]$. The elongation-to-bending ratio converges to a maximum value for an optimum radius of curvature $R$. According to our finite-element simulations, the optimal radius of curvature $R$ for our crystalline resonators is $30 \mu \mathrm{m}$, predicting a $Q$ about four times larger than that obtained with rigid clamping $(R=0)$.

The fabrication of crystalline trampoline resonators introduces technical hurdles that are not present during the fabrication of highly stressed amorphous trampolines $[10,11]$. One major difference is that the thin-film growth of amorphous materials is independent of the crystal orientation of the substrate, while that of crystalline thin films, which are often seeded by the crystal orientation of the substrate, is dependent on the crystal orientation. The crystal-lattice mismatch between the thin film and the seeding substrate induces a high density of crystal defects (a)

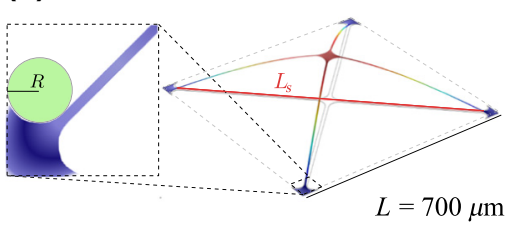

(b)
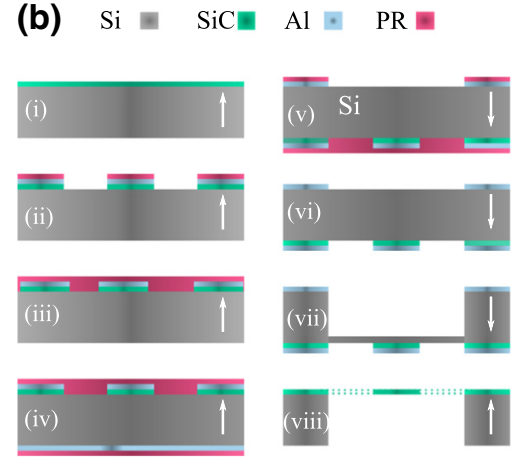

(e)

(c)

(d)
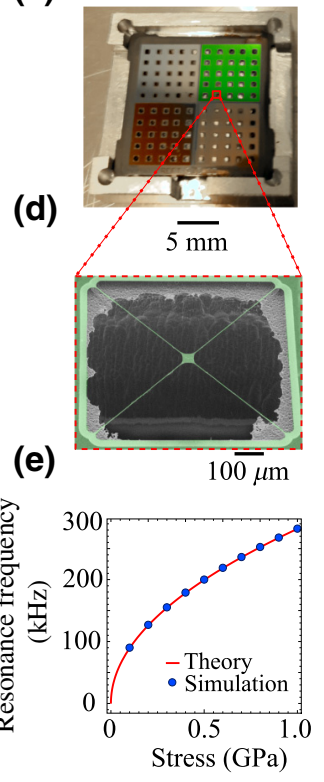

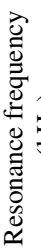

FIG. 1. (a) Mode shape of the fundamental vibrational outof-plane mode of a trampoline with lateral length $L=700 \mu \mathrm{m}$ and clamping points rounded with a radius $R=30 \mu \mathrm{m}$ (inset). (b) Fabrication steps for single-crystal $\mathrm{SiC}$ trampoline resonators. (c) Image of a SiC-on-Si trampoline chip sitting on an aluminum holder. The different colors of the four different chip sections are produced by different thicknesses of the SiC. Each device is released on a fully etched silicon window, which can be directly observed in the image. (d) SEM image of a $\mathrm{SiC}$ trampoline resonator. The trampoline is suspended above a fully back-sideetched hole in a 0.5 -mm-thick silicon substrate. (e) Resonance frequency of a trampoline calculated numerically using finiteelement modeling (blue dots), compared with the resonance frequency of a string of length $L_{s}=\sqrt{2} L$ calculated analytically (red line) using Eq. (2) for a thickness $h=337 \mathrm{~nm}$.

in the first few nanometers of the film, which are accessible by a back-side etch. Release methods used for amorphous materials on silicon such as isotropic wet etching become ineffective for some crystalline films, as the etch rate is strongly dependent on the crystal orientation of the silicon. For this reason, we develop an alternative back-side etch technique for the release of crystalline resonators that is compatible with all crystal orientations of the Si substrate.

Our trampoline resonators are fabricated from a highly stressed 3C-SiC single-crystal thin film developed at the Queensland Microtechnology Facility, in the Queensland node of the Australian National Fabrication Facility. The $\mathrm{SiC}$ material, of initial thickness $h=337 \mathrm{~nm}$, is grown by heteroepitaxial deposition atop a $500-\mu \mathrm{m}$-thick Si substrate, as depicted in Fig. 1(b-i). Using standard photolithography, the trampolines are first patterned on an aluminum thin film $(150 \mathrm{~nm})$ that is evaporated atop the SiC. The wet-etched aluminum functions as a hard mask and as a protective layer during handling. The 
pattern is transferred to the $\mathrm{SiC}$ by reactive ion etching (RIE); see Fig. 1(b-ii). To protect the patterned aluminum and $\mathrm{SiC}$ during later manipulation, a layer of photoresist is spin coated, as illustrated in Fig. 1(b-iii). A $150-\mathrm{nm}$ film of aluminum is evaporated on the back side, which is used as a hard mask during the back-side etch, and the aluminum film is protected with a positive photoresist [Fig. 1(b-iv)]. The front- and back-side patterns, with their corresponding alignment marks, are aligned with the front and rear optical-microscope objectives of an EVG620 mask aligner. The resist is patterned to define square windows, which are transferred to the aluminum using wet etching [Fig. 1(b-v)]. The wafer is placed face down on a secondary carrier wafer, with a thin coating of Fomblin oil between them to enhance thermal contact [Fig. 1(b-vi)]. The silicon is back-side deep-etched by approximately $480 \mu \mathrm{m}$ using deep reactive ion etching, as represented in Fig. 1(b-vii). The chip is then immersed in a heated $\left(80^{\circ} \mathrm{C}\right)$ potassium hydroxide solution to remove the aluminum mask and the excess of silicon "grass" formed during the etching process [39].

To elucidate the effect of the interface crystal defects in the released $\mathrm{SiC}$ structures on the mechanical $Q$, a selective front-side etch is used to vary the thickness $h$ of the $\mathrm{SiC}$ film. The front-side thinning is performed by masking the chip in sections and etching the $\mathrm{SiC}$ layer using RIE dry etching. An image of the chip after etching is presented in Fig. 1(c), where the evident change in color for different film thicknesses is produced by a thin-film interference effect of the illumination light. The trampolines are released using a $\mathrm{XeF}_{2}$ chemical dry etch of the silicon; see Fig. 1(b-viii). As an example, Fig. 1(d) shows a SEM image of a fully released trampoline resonator. The layer with a high density of crystal defects near the $\mathrm{Si}-\mathrm{SiC}$ interface $(>50 \mathrm{~nm})[40]$ becomes accessible once the trampolines are fully released. The chip is flipped and placed on a secondary substrate, and $77 \mathrm{~nm}$ of $\mathrm{SiC}$ is removed from the interface in some trampolines using RIE dry back-side etching, eliminating most of the defect-rich layer.

\section{EXPERIMENTAL RESULTS}

The resonance frequency of highly stressed resonators is strongly dependent on their internal mean stress $\sigma$ and scales as $\sqrt{\sigma}$. The nonuniform residual stress $\sigma_{r}$ in heteroepitaxially grown $3 \mathrm{C}-\mathrm{SiC}$ [29] allows us to investigate the dependence of the mean stress on the film thickness, $\sigma(h)$. To find the relation $\sigma(h)$, we measure the fundamental resonance frequency $\omega_{m}$ of each fabricated trampoline from its noise power spectral density with an optical heterodyne detection system operating in vacuum $\left(P \sim 10^{-6}\right.$ mbar $)$, as reported previously [37,41]. The quality factor $Q=\omega_{m} / \Gamma$ is measured via ringdown after applying an impulse to the trampoline and measuring the
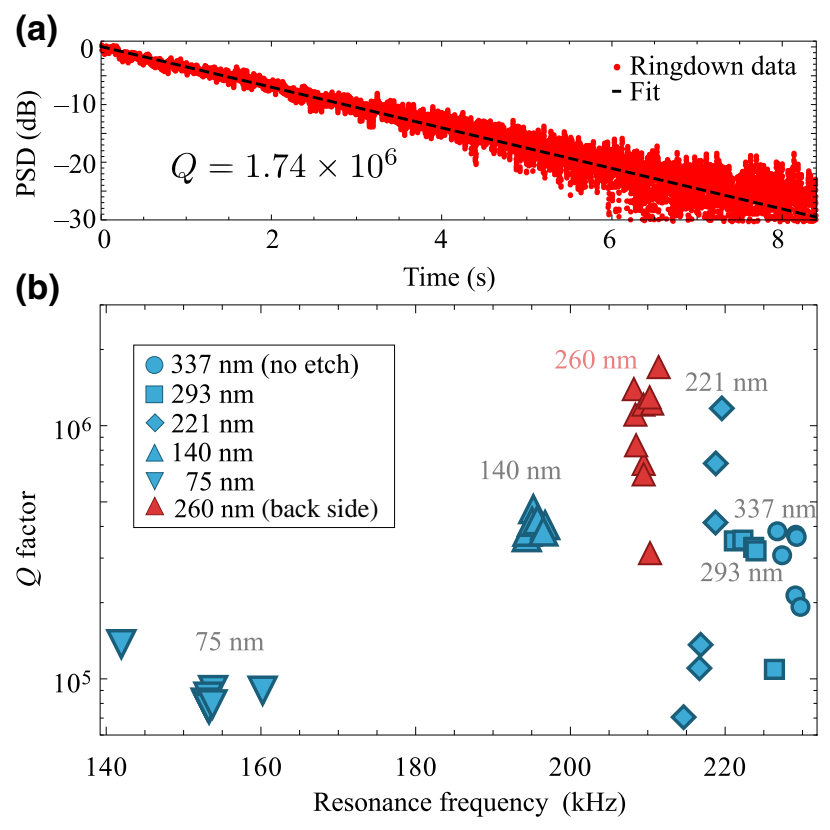

FIG. 2. (a) Normalized ringdown measurement of the fundamental mode of a trampoline resonator with resonance frequency $\omega_{m} / 2 \pi \sim 211 \mathrm{kHz}$ and mechanical quality factor $Q=1.7 \times$ $10^{6}$. Ringdown fitting (black dashed line) using linear regression of the free-ringdown signal captured in a single shot. PSD, power spectral density. (b) Measured resonance frequencies $\omega_{m} / 2 \pi$ and quality factors $Q$ of various single-crystal $\mathrm{SiC}$ trampoline resonators. The blue color code is for front-side-etched devices. Each point represents an individual device, and different shapes correspond to different thicknesses: circles, $h=337 \mathrm{~nm}$; squares, $h=293 \mathrm{~nm}$; rhombuses, $h=221 \mathrm{~nm}$; triangles pointing up, $h=140 \mathrm{~nm}$; and triangles pointing down, $h=75 \mathrm{~nm}$. The red triangles represent the devices that are back-side etched, with a total thickness $h=260 \mathrm{~nm}$.

power decay as $e^{-\Gamma t}$, with a decay rate $\Gamma$. An example of a ringdown measurement (red dots) is shown in Fig. 2(a) for the fundamental mode of a back-side-etched trampoline of frequency $\omega_{m} / 2 \pi=211 \mathrm{kHz}$. The fit (black dashed line) is done using a linear regression of the free-ringdown signal, and we obtain $\Gamma / 2 \pi \approx 1 /(8.23 \mathrm{~s})$. The measured $Q \approx$ $1.7 \times 10^{6}$ for the $\mathrm{SiC}$ trampolines is comparable to the $Q>10^{6}$ achieved in $\mathrm{SiC}$ strings [26]. Moreover, it compares favorably with the $Q \sim 10^{5}$ measured for $\mathrm{Ga}(\mathrm{N}, \mathrm{As})$ crystalline string resonators at room temperature [25], and is comparable to the $Q \sim 2 \times 10^{6}$ for $\mathrm{In}_{x} \mathrm{Ga}_{1-x} \mathrm{P}$ and $\mathrm{GaAs}$ crystalline membrane resonators of similar dimensions $(L \sim 1 \mathrm{~mm})[27,28]$.

The fundamental resonance frequency $\omega_{m}$ and quality factor $Q$ are measured on a total of 45 devices. The results are shown in Fig. 2(b), where each data point represents an individual device. Nine devices, identified by red triangles, are back-side etched by $77 \mathrm{~nm}$, removing the defect-rich layer and leaving a final thickness of $h=260 \mathrm{~nm}$. Six devices with the original film thickness, $h=337 \mathrm{~nm}$ (circles), are measured without etching. The 
other 30 devices are front-side etched, with final thicknesses $h=293 \mathrm{~nm}$ (squares), $h=221 \mathrm{~nm}$ (rhombuses), $h=140 \mathrm{~nm}$ (triangles pointing up), and $h=75 \mathrm{~nm}$ (triangles pointing down). Most of the measured devices have resonance frequencies $\omega_{m} / 2 \pi \gtrsim 200 \mathrm{kHz}$. However, the devices that are greatly front-side etched to a final thickness $h=75 \mathrm{~nm}$ suffer from a significant decrease in both the resonance frequency and the quality factor. This dramatic decrease in $\omega_{m}$ and $Q$ is caused by a substantial reduction of the mean stress $\sigma(h)$, and is consistent with observations that the layer near the interface is under compressive stress [33].

\section{MODEL AND DISCUSSION}

The front- and back-side $\mathrm{SiC}$ etches are expected to have different effects on the $Q$ and resonance frequency of the crystalline resonators due to the presence of crystal defects near the interface. In order to better understand and quantify the role these crystal defects play in the dissipation of crystalline resonators, we develop a generic model for the total $Q$ that allows us to identify five different energydissipation mechanisms, grouped into two main categories, intrinsic and external. The external energy dissipation is attributed to two main mechanisms, gas damping $Q_{\text {gas }}^{-1}$ and clamping losses $Q_{\text {clamp }}^{-1}$ [36]. Our resonators are characterized in a vacuum chamber that operates at $P \sim 10^{-6} \mathrm{mbar}$ and room temperature, with a characteristic Knudsen number $\mathrm{Kn} \sim 75000$, deeply inside the molecular regime $(\mathrm{Kn}>1)$ [36]. This rarefied-gas environment allows us to neglect the damping caused by collisions between gas molecules and the resonator, leaving clamping losses as the main mechanism of external dissipation. These are detailed in Sec. IV B.

Meanwhile, the intrinsic dissipation $Q_{\mathrm{int}}^{-1}=Q_{\mathrm{vol}}^{-1}+Q_{\text {surf }}^{-1}$ is caused by surface losses $\left(Q_{\text {surf }}^{-1}\right)$ [22] and intrinsic friction in the volume of the material $\left(Q_{\mathrm{vol}}^{-1}\right)$ [42], and is detailed in Sec. IV C. Thermoelastic losses in highly stressed trampoline resonators are calculated $\left(Q_{\mathrm{TED}} \sim 10^{9}\right.$ for thermoelastic damping) [37] using existing models [43], and are neglected in the rest of this paper due to their small contribution in thin resonators [44]. The total quality factor is given by

$$
Q^{-1}=\mathcal{D}^{-1} Q_{\text {int }}^{-1}+Q_{\text {clamp }}^{-1},
$$

where $\mathcal{D}(h)$ is the dilution factor and is well approximated by the analytic expression for a string, $\mathcal{D}^{-1}(h) \approx(2 \lambda+$ $\left.\pi^{2} \lambda^{2}\right)$, where $\lambda=(h / L) \sqrt{E / 12 \sigma(h)}$ and $L$ is the length of the string [36]. In Fig. 3, we plot the mean values of the experimentally measured $Q$ (blue squares) and a theoretical fit following Eq. (1) (blue line). In the remainder of the paper, we discuss in detail the model developed to fit $Q$ as a function of thickness for a nonuniform stressed crystalline film.

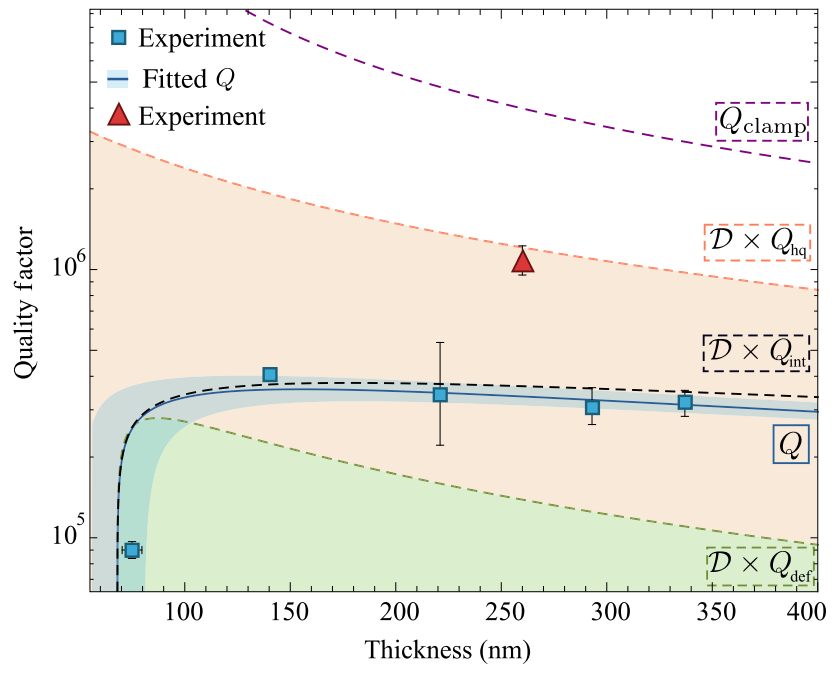

FIG. 3. Mean values of the measured $Q$ of $\mathrm{SiC}$ trampolines with different thicknesses $h$, obtained from the raw data presented in Fig. 2(b). The blue squares (red triangles) are for the frontside- (back-side)-thinned resonators, and the error bars show the standard error. The blue line shows a theoretical fit for $Q$ using Eq. (1) for front-side-etched resonators, and the shaded region shows the uncertainty of the fit. The mean $Q$ for the back-sideetched resonators is shown by a red triangle. The green (orange) dashed line shows $\mathcal{D}(h) \times Q_{\text {def }}\left(\mathcal{D}(h) \times Q_{\text {hq }}\right)$, the limit for trampoline resonators made solely from the defect-rich (high-quality) $\mathrm{SiC}$ layer. The purple dashed line shows $Q_{\text {clamp }}$ calculated from Eq. (4) for a $\mathrm{SiC}$ trampoline resonator with intrinsic stress $\sigma(h)$. The black dashed line shows the diluted intrinsic quality factor $\mathcal{D}(h) \times Q_{\text {int }}$ when clamping losses are neglected.

\section{A. Stress profile and dissipation dilution}

Quantifying $\mathcal{D}(h), Q_{\text {int }}$, and $Q_{\text {clamp }}$ in Eq. (1) requires us to identify the resonance frequency $\omega_{m}(\sigma)$ and the thickness-dependent mean stress $\sigma(h)$. Front-side-thinned resonators experience a reduction of the mean stress $\sigma(h)$, shown in Fig. 4(a) by blue squares. The monotonic decrement of the stress as a function of $h$ is a direct consequence of the declining proportion of high-stress material in the thinner structures. The supplier specifies the mean stress in theSiC film prior to any thinning process $(h=337 \mathrm{~nm})$ as $\sigma_{0} \sim 620 \mathrm{MPa}$, shown in Fig. 4(a) by a dashed line, which agrees reasonably well with our measurements, $\sigma_{0} \sim 660 \mathrm{MPa}$.

There is no known exact analytic solution for the resonance frequencies of a trampoline. However, we find that the fundamental resonance frequency can be accurately modeled by that of a string resonator of length $L_{s}=\sqrt{2} L$, corresponding to the diagonal length of the trampoline. This is given by [36]

$$
\omega_{m}(\sigma)=\left(\frac{\pi}{2 L_{s}}\right)^{2} \sqrt{\frac{4 E h^{2}}{3 \rho}} \sqrt{1+\frac{12 \sigma(h) L_{s}^{2}}{E h^{2} \pi^{2}}},
$$




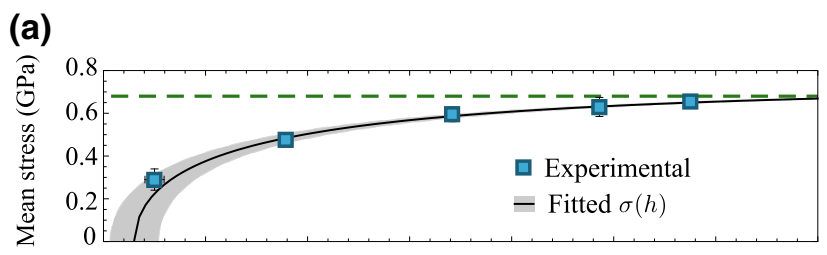

(b)

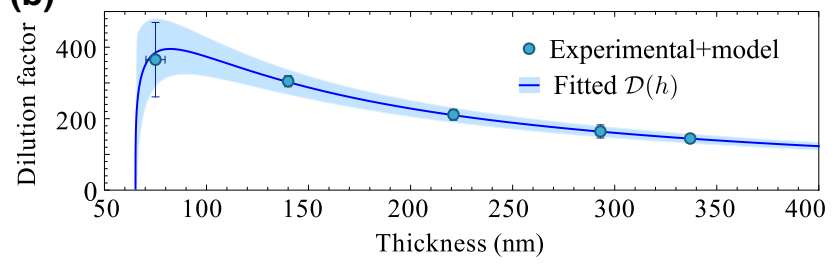

FIG. 4. (a) Mean stress $\sigma(h)$ as a function of the total thickness of the $\mathrm{SiC}$ thin film for front-side-etched resonators. The experimental data are the mean values obtained from the measured resonance frequency using Eq. (3). The error bars represent the standard error. The black line shows the theoretical fit described by Eq. (3), and the gray band shows the associated uncertainty of the fit. (b) Dilution factor $\mathcal{D}(h)$ for a thin-film trampoline with mean stress $\sigma(h)$. The blue circles are determined from the experimental data shown in Fig. 4(a). The light blue band shows the uncertainty of the theoretical fit.

where the density and Young's modulus of SiC are $\rho=$ $3210 \mathrm{~kg} / \mathrm{m}^{3}$ and $E=400 \mathrm{GPa}$, respectively [26]. To confirm the accuracy of this model, the analytic expression (red line) is compared with finite-element modeling (blue dots) for the fundamental vibration mode of the trampoline resonator as a function of the intrinsic mean stress $\sigma$ in the SiC film. As shown in Fig. 1(e), we find very good agreement.

To build an approximate analytical model for $\sigma(h)$ for front-side-thinned resonators, we attempt to fit it to several basic functional forms for growth, including the logistic model [45], Gompertz's model [45], Bridges's growth model [46], and Solow's model [45]. Of these, only Bridges's growth model [46] agrees reasonably well with the mean stress. The final form of the model is

$$
\sigma(h)=\sigma_{\max }\left(1-e^{-\left[c_{1}\left(h-h_{0}\right)\right]^{c_{2}}}\right),
$$

where $h_{0}=(68 \pm 12) \mathrm{nm}$ is the transition thickness at which the mean stress goes from compressive to tensile (i.e., $\sigma\left(h_{0}\right)=0$ ). The exponential growth constant is $c_{1}=0.015 \mathrm{~nm}^{-1}$, and $c_{2}=0.54$ is known as the kinetic order. Using this model, the predicted maximum stress in the high-quality $\mathrm{SiC}$ layer is enhanced to $\sigma_{\max }=740 \mathrm{MPa}$ when there is no defect-rich layer.

From the mean stress $\sigma(h)$, it is also possible to estimate the functional form of the layer-by-layer residual stress $\sigma_{r}(z)$. The residual stress is produced during the heteroepitaxial growth of the $3 \mathrm{C}-\mathrm{SiC}$ thin films and is related to the mean stress in the film through the relation $\sigma(h)=(1 / h) \int_{0}^{h} \sigma_{r}(z) d z$. So far, its functional form has not been well known, because existing spectroscopic methods for measuring $\sigma_{r}(z)$ are incompatible with thin-film analysis. For example, Raman spectroscopy suffers from limited resolution (approximately $1 \mu \mathrm{m}$ ) [47], while other measurement methods such as bulge testing are incompatible with micro- and nanomechanical systems as they are limited to centimeter-scale sealed membranes [33]. Our method of characterizing the mean stress in released microstructures could in future provide a precise determination of the residual-stress profile $\sigma_{r}(z)$ of heteroepitaxial thin films beyond the resolution of existing noninvasive methods $[33,47]$.

The experimental $\mathcal{D}(h)$ shown in Fig. 4(b) (blue points) is determined by combining the measurements of the stress $\sigma(h)$ [see Fig. 4(a)] and the analytical model of the thickness-dependent dilution factor $\mathcal{D}(h)$ for a string of length $L_{s}$ [36]. The good agreement provides experimental validation, relevant both to our work and to previous research $[11,35,38]$, that it is appropriate to model trampolines as strings. The combined results in Fig. 4 suggest that the highest enhancement of the quality factor should occur at a film thickness $h$ of approximately $70 \mathrm{~nm}$. However, the total $Q$ is affected not just by the dilution factor but also by intrinsic loss mechanisms and clamping losses.

For highly stressed resonators, clamping losses are often neglected as the largest contribution to the loss comes from intrinsic mechanisms. In this limit, the intrinsic quality factor $Q_{\text {int }}$ is estimated by dividing $Q \approx \mathcal{D}(h) \times Q_{\text {int }}$ (black dashed line in Fig. 3) by the dilution factor $\mathcal{D}(h)$. However, for our trampolines we find that the experimental results deviate from this model at large thicknesses, as shown in Fig. 3 (blue line), indicating that clamping losses should be included. We expand our model further for clamping losses in Sec. IV B and for intrinsic losses in Sec. IV C.

\section{B. Clamping loss}

Clamping losses are caused by phonons tunneling from the resonator into the substrate [48]. Elastic energy leaks out of the resonator through the clamping points in the form of acoustic radiation. The amount of leakage depends on the impedance mismatch between the resonator and the substrate [36]. To estimate the clamping losses produced in our system, we use finite-element modeling (FEM) to calculate the total elastic energy $U$ stored in the resonator and the power $P_{\text {acou }}$ carried by the acoustic radiation. The clamping-loss-dominated quality factor $Q_{\text {clamp }}$ can be estimated from the ratio of the energy stored to the energy lost during one oscillation cycle as $Q_{\text {clamp }}=2 \pi \omega_{m}\left(U /\left\langle P_{\text {acou }}\right\rangle\right)$. In Fig. 5(a), we show a calculation of $P_{\text {acou }}$ for a trampoline attached to a substrate of thickness $h_{s}=500 \mu \mathrm{m}$, where symmetries are used to reduce the computational demand by calculating over one quarter of the domain. The trampoline design is represented in Fig. 5(b), where the 
(a)
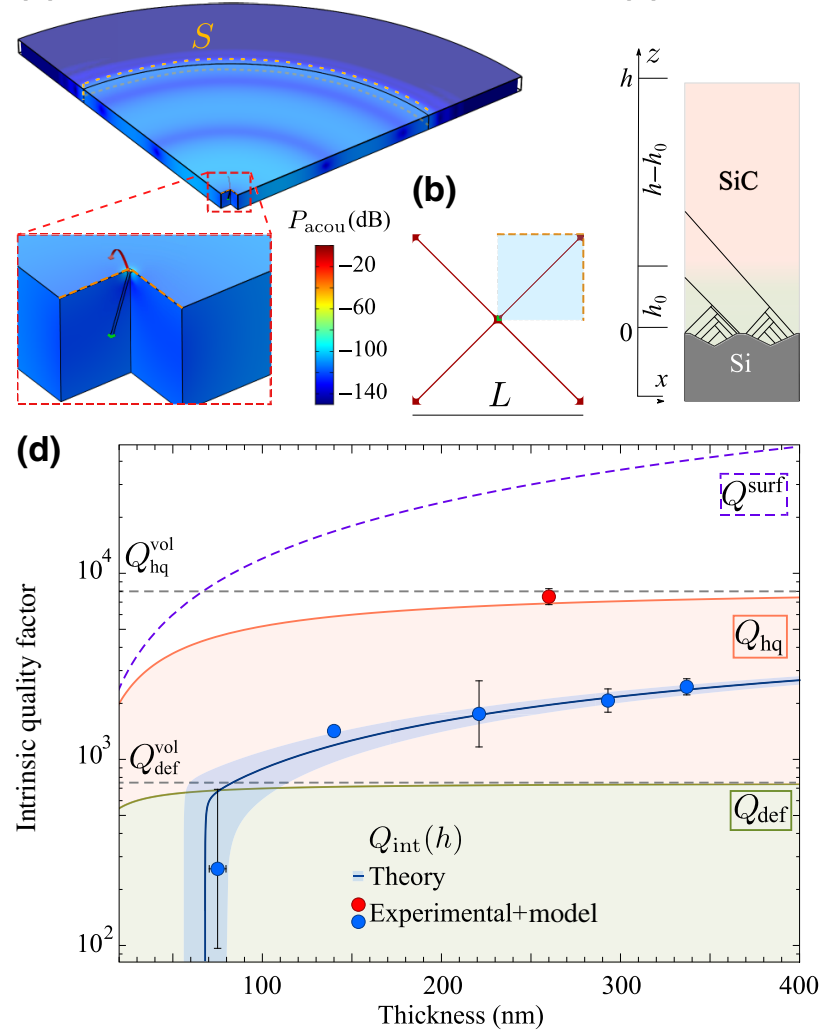

FIG. 5. (a) FEM simulation of the acoustic power $P_{\text {acou }}$ propagating through the $\mathrm{Si}$ substrate, with a perfectly matched layer at the surface $S$, calculated in a quarter of the total domain using symmetries, with enlargement of the quarter of the trampoline used in the calculation. (b) Top view of the trampoline design, where the blue-shaded region represents the domain used in the FEM simulation. The clamped boundaries, shown by colored lines, are mapped into the substrate for reference. (c) Schematic lateral view of the $\mathrm{SiC}$ single crystal atop $\mathrm{Si}$, based on TEM studies and images $[33,49]$. The region labeled $h_{0}$ represents the thickness of the defect-rich layer localized near the interface. The lines near the interface illustrate stacking defects and dislocations. (d) Thickness-dependent fitting parameters for the bilayer system: the intrinsic parameter $Q_{\text {def }}$ of the defect-rich layer (green line) and that of the high-quality single crystal, $Q_{\mathrm{hq}}$ (orange line), and the thickness-dependent surface loss $Q^{\text {surf }}(h)$ (purple dashed line) of the bilayer system. Each layer is limited by its respective volume loss $Q_{\mathrm{vol}}^{\mathrm{hq} \text {,def }}$ (gray dashed lines). A theoretical fit of $Q_{\text {int }}$ obtained from Eq. (5) for the front-sideetched resonators is compared with the experimental data, with the blue-shaded region being the uncertainty of the fit.

blue-shaded region is the quarter of the domain used during the calculations. The power crossing the interface $S$ leaves the substrate, where a perfectly matched layer attenuates it and prevents it from being reflected. The parameters used in the FEM calculation include the Young's modulus of the $\mathrm{Si}$ substrate, $E_{s}=170 \mathrm{GPa}$, and the density, $\rho_{s}=2650 \mathrm{~kg} / \mathrm{m}^{3}[26]$.
The numerical results obtained for $Q_{\text {clamp }}$ from the FEM simulations require substantial computational time and fail to predict the clamping losses at small thickness, where an analytic model presents an advantage. Trampoline resonators are two-dimensional structures that have similarities with membrane resonators, for which an analytic expression,

$$
Q_{\text {clamp }}=\alpha \frac{3 \rho_{s}}{2 \rho} \sqrt{\frac{E_{s} \rho}{2 \sigma(h) \rho_{s}}} \frac{L}{h},
$$

exists for the fundamental mode. Comparing our numerical solution with this analytic expression, we find agreement for a prefactor $\alpha=200$, which is a fitting parameter correcting for substrate imperfections and mounting conditions [22]. Consequently, the analytic model is used henceforth. With $\alpha$ fitted, the results obtained from the FEM simulations and the analytic expressions agree to within $0.01 \%$ for $h \geq 200 \mathrm{~nm}$ and to within approximately $5 \%$ for $h \sim 100 \mathrm{~nm}$, diverging for thinner thicknesses due to limitations on the meshing of the domain of the FEM simulation. In Fig. 3, we plot $Q_{\text {clamp }}$ (purple dashed line) as a function of thickness, showing that it is not the primary limitation on the performance of our resonators, but cannot be entirely neglected.

\section{Intrinsic dissipation}

The intrinsic dissipation $\left(Q_{\text {int }}^{-1}\right)$ in microresonators originates from two main sources, surface losses $\left(Q_{\text {surf }}^{-1}\right)$ and volume losses $\left(Q_{\mathrm{vol}}^{-1}\right)$. The dominant contribution to the surface losses is expected to occur at the top and the bottom surfaces of the device, since these have a much larger area than the lateral surfaces [22]. The volume losses in crystalline resonators are caused by defect motion in the resonator volume [34]. In materials with a nonuniform vertical density of defects, such as heteroepitaxially grown $3 \mathrm{C}-\mathrm{SiC}$, the dissipation profile is expected to be vertically nonuniform.

As a simple model of the nonuniform distribution of defects, we consider the $\mathrm{SiC}$ film as a bilayer system. The first layer is a defect-rich layer (def) near the $\mathrm{Si}-\mathrm{SiC}$ interface with a thickness $h_{0}$, as represented in Fig. 5(c). The second layer is a high-quality single-crystal layer (hq) with a thickness $h-h_{0}$, above the defect-rich layer; it is expected to have a significantly reduced density of defects (shown by black lines), but defects will not be eliminated [40]. In order to identify the different contributions to the intrinsic dissipation in this bilayer system, two main assumptions are made: first, that each layer has independent volume and surface dissipation components, and second, that the surface dissipation follows an inverse linear relation with the thickness, as has been shown for silicon nitride membranes and microcantilevers $[22,44]$. For a high-quality crystal $\left(h>h_{0}\right)$, the surface loss is then 
given by $Q_{\mathrm{hq}}^{\text {surf }}(h)=\beta_{\mathrm{hq}}\left(h-h_{0}\right)$ for the high-quality layer, and $Q_{\text {def }}^{\text {surf }}=\beta_{\text {def }} \times h_{0}$ for the defect-rich layer.

The total intrinsic dissipation $Q_{\text {int }}^{-1}(h)$ is given by a weighted sum of the dissipations in each layer,

$$
\begin{aligned}
Q_{\text {int }}^{-1}(h)= & \left(\frac{h-h_{0}}{h}\right)\left\{\left[Q_{\mathrm{hq}}^{\text {surf }}(h)\right]^{-1}+\left(Q_{\mathrm{hq}}^{\text {vol }}\right)^{-1}\right\} \\
& +\left(\frac{h_{0}}{h}\right)\left[\left(Q_{\text {def }}^{\text {surf }}\right)^{-1}+\left(Q_{\text {def }}^{\text {vol }}\right)^{-1}\right],
\end{aligned}
$$

where $Q_{\mathrm{hq}}^{\mathrm{vol}}$ and $Q_{\mathrm{def}}^{\mathrm{vol}}$ are the volume quality factors of the high-quality crystal and the defect-rich layer, respectively. The experimentally determined values of $Q_{\text {int }}$ (blue dots) shown in Fig. 5(d) are deduced from Eq. (1) by combining the experimental $Q$ presented in Fig. 3 with the analytic models for $Q_{\text {clamp }}$ and $\mathcal{D}(h)$. Meanwhile, the theoretical fit is obtained from simultaneously fitting the five parameters of the bilayer $\mathrm{SiC}$ model $Q_{\mathrm{def}}^{\mathrm{vol}}$, $Q_{\mathrm{hq}}^{\mathrm{vol}}, \beta_{\mathrm{hq}}, \beta_{\mathrm{def}}$, and $\alpha$ to the six data points in Fig. 5(c). The total surface loss in the bilayer model is given by $Q^{\text {surf }}(h)=\left[\left(h-h_{0} / h\right)\left[Q_{\mathrm{hq}}^{\text {surf }}(h)\right]^{-1}+\left(h_{0} / h\right)\left(Q_{\mathrm{def}}^{\text {surf }}\right)^{-1}\right]^{-1}$, and is shown as a function of film thickness (blue dashed line). The fitting parameters are summarized in Table I.

Volumetric dissipation is associated with friction acting on crystal dislocations, stacking faults, or defects in the bulk SiC. Accordingly, the dissipation is expected to be higher in regions of the film with a high defect density than in regions with a low defect density. Our fitting parameters $Q_{\mathrm{hq}}^{\mathrm{vol}}=(8.0 \pm 1.8) \times 10^{3}$ and $Q_{\mathrm{def}}^{\mathrm{vol}}=750 \pm 150$ are depicted as gray dashed lines in Fig. 5(d), where the green and orange lines illustrate the intrinsic quality factors for trampolines made solely from the defect-rich layer and the high-quality layer, respectively. The fact that $Q_{\mathrm{hq}}^{\mathrm{vol}}$ is more than an order of magnitude higher than $Q_{\text {def }}^{\mathrm{vol}}$ confirms that the defect layer does indeed have a significantly degraded quality factor due to crystal defects at the interface and that these defects increase the intrinsic dissipation.

The surface losses of the defect and high-quality layers, $\beta_{\text {def }}=(10 \pm 4) \times 10^{10} \mathrm{~m}^{-1}$ and $\beta_{\mathrm{hq}}=(12 \pm 5) \times$ $10^{10} \mathrm{~m}^{-1}$, respectively, are identical within the uncertainty of the fit. This suggests that the surface losses are not

TABLE I. Volume and surface quality factors of the defect-rich and high-quality layers.

\begin{tabular}{cc}
\hline \hline Parameter & Value \\
\hline$Q_{\text {def }}^{\text {vol }}$ & $(0.75 \pm 0.15) \times 10^{3}$ \\
$Q_{\text {def }}^{\text {surf }}$ & $(10 \pm 4) \times 10^{10} \mathrm{~m}^{-1} \times h_{0}$ \\
$Q_{\mathrm{hq}}^{\text {vol }}$ & $(8.0 \pm 1.8) \times 10^{3}$ \\
$Q_{\mathrm{hq}}^{\text {surf }}$ & $(12 \pm 5) \times 10^{10} \mathrm{~m}^{-1} \times\left(h-h_{0}\right)$ \\
$h_{0}$ & $(68 \pm 12) \times 10^{-9} \mathrm{~m}$ \\
\hline \hline
\end{tabular}

drastically affected by the etching process, and that interfacial defects have an impact on the volume component of the dissipation rather than the surface component; this is consistent with the chemical stability of the surface composition of $\mathrm{SiC}$ [50,51].

The highest quality factor, $Q=1.74 \times 10^{6}$, is obtained on a back-side-etched resonator. The average $Q$ of the back-side-etched resonators is about five times higher than the predicted average value for front-side-etched resonators of the same thickness, and more than an order of magnitude higher than the $Q$ measured for the thinnest front-side-etched resonators. This results from an increase in the intrinsic quality factor $Q_{\text {int }}$ by almost one order of magnitude for back-side-etched resonators compared with front-side-etched resonators of similar thickness. The $Q$ values of front- and back-side-etched resonators are limited primarily by their volume losses.

While already comparable with previous results on crystalline resonators $[25,27,28]$, our results show that $\mathrm{SiC}$ resonators have significant possibilities for further improvement. Even though the high-quality single-crystal layer has a low density of defects, these are not eliminated [40]. The volumetric part of the intrinsic quality factor, while it remains well below the single-crystal limit for $\mathrm{SiC}, Q_{\mathrm{hq}}^{\mathrm{vol}} \sim 10^{5}$ [42], compares positively with that of LPCVD silicon nitride resonators that have already reached the volume-loss limit for their amorphous composition $[14,22,38]$. The complete removal of interfacial crystal defects in SiC could therefore potentially lead to exceptional enhancements of the quality factor to values as high as $\mathcal{D}(h) \times Q_{\text {int }} \sim 10^{8}$ for a trampoline of thickness $h=70 \mathrm{~nm}$. Moreover, implementing dissipationengineered designs exploiting the high yield strength of $\mathrm{SiC}$ could allow quality factors as high as $Q \sim 6 \times 10^{9}$ $[15,16]$.

\section{CONCLUSION}

This paper explores the possibility of achieving ultrahigh quality factors in crystalline thin-film microresonators. Our results verify the prediction that interfacial defects in the thin films can severely degrade the intrinsic quality factor of crystalline resonators. We develop a crystalline-material-compatible back-side etch procedure to remove the layer containing interfacial defects, which can be applied to enhance the intrinsic quality factor of other crystalline stressed resonators made from epitaxially grown materials [24,25,27,28].

Our method allows a factor-of-five improvement in the diluted quality factor for single-crystal silicon carbide resonators by increasing the intrinsic quality factor, achieving values of $Q>10^{6}$. By developing a detailed model of the dissipation in bilayer films, we are able to precisely determine the material properties of $\mathrm{SiC}$ epitaxial films with 
higher resolution than can be done with spectroscopic techniques. Our model predicts that diluted quality factors as high as $6 \times 10^{9}$ may be possible using both dissipation and strain engineering if high-quality single-crystal silicon carbide is used.

\section{ACKNOWLEDGMENTS}

This research is funded by the Australian Research Council and Lockheed Martin Corporation through the Australian Research Council Linkage Grant No. LP140100595. This research is partially supported by the Commonwealth of Australia as represented by the Defence Science and Technology Group of the Department of Defence. Support is also provided by the Australian Research Council Centre of Research Excellence for Engineered Quantum Systems (Grant No. CE110001013). E.R. and V.M.V. acknowledge CONACYT (Grants No. 381542 and No. 234733, respectively). V.M.V. acknowledges the Laboratorio Nacional de Materia Cuántica (Grants No. LN-293471 and No. LN-299057). W.P.B. acknowledges a Future Fellowship from the Australian Research Council (Grant No. FT140100650). The 3C-SiC material is developed and supplied by Leonie Hold and Alan Iacopi of the Queensland Microtechnology Facility, in the Queensland node of the Australian National Fabrication Facility. This work is performed in part at the Queensland node of the Australian National Fabrication Facility, a company established under the National Collaborative Research Infrastructure Strategy to provide nano- and microfabrication facilities for Australia's researchers. The authors acknowledge James Bennett for useful discussions and technical support.

[1] Y. T. Yang, C. Callegari, X. L. Feng, K. L. Ekinci, and M. L. Roukes, Zeptogram-scale nanomechanical mass sensing, Nano Lett. 6, 583 (2006).

[2] M. Chien, M. Brameshuber, B. K. Rossboth, G. J. Schütz, and S. Schmid, Single-molecule optical absorption imaging by nanomechanical photothermal sensing, Proc. Natl. Acad. Sci. USA 115, 11150 (2018).

[3] S. Basiri-Esfahani, A. Armin, S. Forstner, and W. P. Bowen, Precision ultrasound sensing on a chip, Nat. Commun. 10, 132 (2019).

[4] B. Li, J. Bílek, U. B. Hoff, L. S. Madsen, S. Forstner, V. Prakash, C. Schäfermeier, T. Gehring, W. P. Bowen, and U. L. Andersen, Quantum enhanced optomechanical magnetometry, Optica 5, 850 (2018).

[5] X. Fan, F. Forsberg, A. D. Smith, S. Schröder, S. Wagner, H. Rödjegård, A. C. Fischer, M. Östling, M. C. Lemme, and F. Niklaus, Graphene ribbons with suspended masses as transducers in ultra-small nanoelectromechanical accelerometers, Nat. Electron. 2, 394 (2019).

[6] A. Vinante, R. Mezzena, P. Falferi, M. Carlesso, and A. Bassi, Improved Noninterferometric Test of Collapse
Models Using Ultracold Cantilevers, Phys. Rev. Lett. 119, 110401 (2017).

[7] S. Forstner, M. Zych, S. Basiri-Esfahani, K. E. Khosla, and W. P. Bowen, arXiv:1909.01608 [quant-ph] (2019).

[8] M. Bagheri, M. Poot, M. Li, W. P. H. Pernice, and H. X. Tang, Dynamic manipulation of nanomechanical resonators in the high-amplitude regime and non-volatile mechanical memory operation, Nat. Nanotech. 6, 726 (2011).

[9] W. Jiang, C. J. Sarabalis, Y. D. Dahmani, R. N. Patel, F. M. Mayor, T. P. McKenna, R. Van Laer, and A. H. SafaviNaeini, Efficient bidirectional piezo-optomechanical transduction between microwave and optical frequency, Nat. Commun. 11, 1166 (2020).

[10] C. Reinhardt, T. Müller, A. Bourassa, and J. C. Sankey, Ultralow-Noise SiN Trampoline Resonators for Sensing and Optomechanics, Phys. Rev. X 6, 021001 (2016).

[11] R. A. Norte, J. P. Moura, and S. Gröblacher, Mechanical Resonators for Quantum Optomechanics Experiments at Room Temperature, Phys. Rev. Lett. 116, 147202 (2016).

[12] Y. Tsaturyan, A. Barg, E. S. Polzik, and A. Schliesser, Ultracoherent nanomechanical resonators via soft clamping and dissipation dilution, Nat. Nano 12, 776 (2017).

[13] M. Rossi, D. Mason, J. Chen, Y. Tsaturyan, and A. Schliesser, Measurement-based quantum control of mechanical motion, Nature 563, 53 (2018).

[14] A. H. Ghadimi, D. J. Wilson, and T. J. Kippenberg, Radiation and internal loss engineering of high-stress silicon nitride nanobeams, Nano Lett. 17, 3501 (2017).

[15] A. H. Ghadimi, S. A. Fedorov, N. J. Engelsen, M. J. Bereyhi, R. Schilling, D. J. Wilson, and T. J. Kippenberg, Elastic strain engineering for ultralow mechanical dissipation, Science 360, 764 (2018).

[16] S. A. Fedorov, N. J. Engelsen, A. H. Ghadimi, M. J. Bereyhi, R. Schilling, D. J. Wilson, and T. J. Kippenberg, Generalized dissipation dilution in strained mechanical resonators, Phys. Rev. B 99, 054107 (2019).

[17] H. Najar, M.-L. Chan, H.-A. Yang, L. Lin, D. G. Cahill, and D. A. Horsley, High quality factor nanocrystalline diamond micromechanical resonators limited by thermoelastic damping, Appl. Phys. Lett. 104, 151903 (2014).

[18] Y. Tao, J. M. Boss, B. A. Moores, and C. L. Degen, Singlecrystal diamond nanomechanical resonators with quality factors exceeding one million, Nat. Commun. 5, 4638 (2014).

[19] J. Hofer, A. Schliesser, and T. J. Kippenberg, Cavity optomechanics with ultrahigh- $Q$ crystalline microresonators, Phys. Rev. A 82, 031804(R) (2010).

[20] B. Jiang, N. Opondo, G. Wolfowicz, P.-L. Yu, D. D. Awschalom, and S. A. Bhave, in 2019 20th International Conference on Solid-State Sensors, Actuators and Microsystems Eurosensors XXXIII (TRANSDUCERS EUROSENSORS XXXIII) (IEEE, Berlin, Germany, 2019), p. 1655, ISSN: 2167-0013.

[21] B. Hamelin, J. Yang, A. Daruwalla, H. Wen, and F. Ayazi, Monocrystalline silicon carbide disk resonators on phononic crystals with ultra-low dissipation bulk acoustic wave modes, Sci. Rep. 9, 18698 (2019).

[22] L. G. Villanueva and S. Schmid, Evidence of Surface Loss as Ubiquitous Limiting Damping Mechanism in SiN Micro- 
and Nanomechanical Resonators, Phys. Rev. Lett. 113, 227201 (2014).

[23] J. Gaspar, V. Chu, and J. P. Conde, Amorphous silicon electrostatic microresonators with high quality factors, Appl. Phys. Lett. 84, 622 (2004).

[24] M. Bückle, V. C. Hauber, G. D. Cole, C. Gärtner, U. Zeimer, J. Grenzer, and E. M. Weig, Stress control of tensile-strained $\operatorname{In}_{1-x} \mathrm{Ga}_{x} \mathrm{P}$ nanomechanical string resonators, Appl. Phys. Lett. 113, 201903 (2018).

[25] K. Onomitsu, M. Mitsuhara, H. Yamamoto, and H. Yamaguchi, Ultrahigh- $Q$ micromechanical resonators by using epitaxially induced tensile strain in GaNAs, Appl. Phys. Express 6, 111201 (2013).

[26] A. R. Kermany, G. Brawley, N. Mishra, E. Sheridan, W. P. Bowen, and F. Iacopi, Microresonators with $Q$-factors over a million from highly stressed epitaxial silicon carbide on silicon, Appl. Phys. Lett. 104, 081901 (2014).

[27] J. Liu, K. Usami, A. Naesby, T. Bagci, E. S. Polzik, P. Lodahl, and S. Stobbe, High- $Q$ optomechanical GaAs nanomembranes, Appl. Phys. Lett. 99, 243102 (2011).

[28] G. D. Cole, P.-L. Yu, C. Gärtner, K. Siquans, R. Moghadas Nia, J. Schmöle, J. Hoelscher-Obermaier, T. P. Purdy, W. Wieczorek, C. A. Regal, and M. Aspelmeyer, Tensilestrained $\mathrm{In}_{x} \mathrm{Ga}_{1-x} \mathrm{P}$ membranes for cavity optomechanics, Appl. Phys. Lett. 104, 201908 (2014).

[29] A. R. Kermany, J. S. Bennett, G. A. Brawley, W. P. Bowen, and F. Iacopi, Factors affecting the fxQ product of $3 \mathrm{C}$ SiC microstrings: What is the upper limit for sensitivity?, J. Appl. Phys. 119, 055304 (2016).

[30] K. E. Petersen, Silicon as a mechanical material, Proc. IEEE 70, 420 (1982).

[31] F. La Via, A. Severino, R. Anzalone, C. Bongiorno, G. Litrico, M. Mauceri, M. Schoeler, P. Schuh, and P. Wellmann, From thin film to bulk $3 \mathrm{C}-\mathrm{SiC}$ growth: Understanding the mechanism of defects reduction, Mater. Sci. Semicond. Process 78, 57 (2018).

[32] R. Anzalone, G. Litrico, N. Piluso, R. Reitano, A. Alberti, P. Fiorenza, S. Coffa, and F. La Via, Carbonization and transition layer effects on 3C-SiC film residual stress, J. Cryst. Growth 473, 11 (2017).

[33] F. Iacopi, R. E. Brock, A. Iacopi, L. Hold, and R. H. Dauskardt, Evidence of a highly compressed nanolayer at the epitaxial silicon carbide interface with silicon, Acta Mater. 61, 6533 (2013).

[34] A. N. Cleland and M. L. Roukes, Noise processes in nanomechanical resonators, J. Appl. Phys. 92, 2758 (2002).

[35] M. J. Bereyhi, A. Beccari, S. A. Fedorov, A. H. Ghadimi, R. Schilling, D. J. Wilson, N. J. Engelsen, and T. J. Kippenberg, Clamp-tapering increases the quality factor of stressed nanobeams, Nano Lett. 19, 2329 (2019).

[36] S. Schmid, L. G. Villanueva, and M. L. Roukes, Fundamentals of Nanomechanical Resonators (Springer International Publishing, Cham, 2016).
[37] E. Romero Sanchez, Ph.D. thesis, The University of Queensland, 2019.

[38] P. Sadeghi, M. Tanzer, S. L. Christensen, and S. Schmid, Influence of clamp-widening on the quality factor of nanomechanical silicon nitride resonators, J. Appl. Phys. 126, 165108 (2019).

[39] K. Jung, S. WooJin, L. Hyun Woo, and C. S. Lee, Parameter study for silicon grass formation in Bosch process, J. Vac. Sci. Technol. 28, 143 (2010).

[40] F. Iacopi, G. Walker, L. Wang, L. Malesys, S. Ma, B. V. Cunning, and A. Iacopi, Orientation-dependent stress relaxation in hetero-epitaxial 3C-SiC films, Appl. Phys. Lett. 102, 011908 (2013).

[41] E. Romero, R. Kalra, N. P. Mauranyapin, C. G. Baker, C. Meng, and W. P. Bowen, Propagation and Imaging of Mechanical Waves in a Highly Stressed SingleMode Acoustic Waveguide, Phys. Rev. Appl. 11, 064035 (2019).

[42] M. F. Ashby, Overview No. 80: On the engineering properties of materials, Acta Metall. 37, 1273 (1989).

[43] R. Lifshitz and M. L. Roukes, Thermoelastic damping in micro- and nanomechanical systems, Phys. Rev. B 61, 5600 (2000).

[44] K. Y. Yasumura, T. D. Stowe, E. M. Chow, T. Pfafman, T. W. Kenny, B. C. Stipe, and D. Rugar, Quality factors in micron- and submicron-thick cantilevers, J. Microelectromech. Syst. 9, 117 (2000).

[45] M. J. Panik, Growth Curve Modeling: Theory and Applications (John Wiley \& Sons, Hoboken, New Jersey, 2014).

[46] T. C. Bridges, L. W. Turner, E. M. Smith, T. S. Stahly, and O. J. Loewer, A mathematical procedure for estimating animal growth and body composition, Trans. ASAE 29, 1342 (1986).

[47] R. Anzalone, G. D'Arrigo, M. Camarda, C. Locke, S. E. Saddow, and F. L. Via, Advanced residual stress analysis and FEM simulation on heteroepitaxial 3C-SiC for MEMS application, J. Microelectromech. Syst. 20, 745 (2011).

[48] G. D. Cole, I. Wilson-Rae, K. Werbach, M. R. Vanner, and M. Aspelmeyer, Phonon-tunnelling dissipation in mechanical resonators, Nat. Commun. 2, 231 (2011).

[49] Y. Sun, S. Izumi, S. Sakai, K. Yagi, and H. Nagasawa, Saddle-shape warpage of thick 3C-SiC wafer: Effect of nonuniform intrinsic stress and stacking faults, Phys. Status Solidi B 249, 555 (2012).

[50] M. Mehregany, C. A. Zorman, and N. R. and, Silicon carbide MEMS for harsh environments, Proc. IEEE 86, 1594 (1998).

[51] R. G. Azevedo, D. G. Jones, A. V. Jog, B. Jamshidi, D. R. Myers, L. Chen, X. Fu, M. Mehregany, M. B. J. Wijesundara, and A. P. Pisano, A SiC MEMS resonant strain sensor for Harsh environment applications, IEEE Sens. J. 7, 568 (2007). 